\title{
Epitopes prediction of PfEMP1-DBL2 $\beta$ recombinant protein from Indonesian Plasmodium falciparum Isolate for malaria vaccine development
}

\author{
Sheilla Rachmania ${ }^{1,2}$, Erma Sulistyaningsih ${ }^{1,2,3}$, Anak Agung Istri Ratnadewi ${ }^{1,3,4}$, Rosita Dewi ${ }^{1,2}$ \\ ${ }^{1}$ Graduate School of Biotechnology, University of Jember, ${ }^{2}$ Faculty of Medicine, University of Jember, ${ }^{3}$ Center for \\ Development of Advance Science and Technology (CDAST), University of Jember, ${ }^{4}$ Faculty of Mathematics and \\ Natural Sciences, University of Jember
}

\begin{abstract}
The development of an effective vaccine against malaria is essential. Domain Duffy-binding-like (DBL)2 $\beta$ in Plasmodium falciparum erythrocyte membrane protein 1 (PfEMP1) protein is one of the vaccine candidate proteins because of its binding capacity to ICAM-1 receptor. However, the high polymorphism needs epitope identification before formulating a peptide-based vaccine. This study aimed to identify the conserved epitopes in PfEMP1-DBL2 $\beta$ by an immunoinformatic approach. The protein sequences were analyzed to predict the hydrophobicity attributes, T-cell and B-cell epitopes. T-cell epitopes were identified using the NetCTL and Propred server and analyzed for population coverage rate using the IEDB analysis tool. Moreover, the Bepipred 2.0 and Kolaskar Tangaonkar method combined with the K-means clustering were used to predict the B-cell epitopes. This study found that the hydrophobicity value of PfEMP1-DBL2 $\beta$ recombinant protein is 32.62 , indicating that this protein is soluble and potentially fit into HLA alleles active site. Two conserved antigenic CTL epitopes with near $90 \%$ population coverage rate in the malaria target population were identified. For Th cell epitopes, the $\mathrm{NN}$-align algorithm showed no overlapping strong binding epitope positions for three chosen Indonesian and African alleles. Three B-cell conserved epitopes were identified at the position of 77-89, 236-254 and 360-377 amino acids with one cluster overlapping with ICAM-1 determinant binding area. The predicted conserved epitopes within the protein in this study are valuable in constructing a subunit peptide-based malaria vaccine candidate. A further experimental study is needed to validate this approach as the next step in vaccine development.
\end{abstract}

Keywords: DBL2 $\beta$, epitope, immunoinformatic, malaria, PfEMP1.

\section{Introduction}

Malaria is one of the most important infectious diseases; it is responsible for 400.000 deaths each year worldwide. Indonesia is one of the countries that

\section{Corresponding Author:}

\section{Dr. Rer. Biol. Hum. Dr. Erma Sulistyaningsih}

M.Si, Department of Parasitology, Faculty of Medicine, University of Jember, Jember, East Java, Indonesia

68121

Phone: +62331337877

e-mail:sulistyaningsih.fk@unej.ac.id have more than 100.000 increasing cases in $2017^{(1)}$. WHO has several strategies and recommendations to control malaria. A vaccination is considered a great and effective solution as a prophylactic treatment for infectious disease, especially for severe malaria-causing high morbidity and mortality ${ }^{(2)}$.

Pathology of severe malaria involves aggregation and adhesion in microvascular of vital organs called cytoadherence. This mechanism reduces and further obstructs blood flow resulting in organ failure. Besides, cytoadherence can contribute to immune evasion mechanisms causing the spleen's inability to destruct the infected erythrocyte ${ }^{(3)}$. The cytoadherence is mediated 
by Plasmodium falciparum erythrocyte membrane protein-1 (PfEMP1), a protein expressed by the parasite and deposited on the surface of infected erythrocyte which can bind to various host cell receptors. PfEMP1 is a complex protein and consists of several domains, one of them is the Duffy-binding-like (DBL) $2 \beta$ domain that has an affinity toward Intercellular Adhesion Molecule-1 (ICAM-1) receptor found in endothelial cell of microvasculature including the brain ${ }^{(4)}$.

The adhesion of PfEMP1 domains to several host receptors plays an essential role in mediating severe malaria pathogenesis, thus makes this protein as a target in developing the peptide-based vaccine. However, the complexity of the parasite's life cycle, high antigenic switching rate and immune evasion ability of the parasites are the major problems ${ }^{(5)}$.

The development of the current vaccine is focusing on the discovery of subunit epitope-based vaccines that incorporate one or more semi-purified or even purified antigens. To develop a subunit vaccine, determine the immunological properties of the target protein in generating protection is critical since some peptides may be immunosuppressive or even enhance the disease. Peptides associated with protective epitopes are preferable vaccine candidates, but testing out every peptide as an epitope for their ability to generate protective immune response has several limitations. Aside from time and labor consuming, most peptides expressed during in vivo infection is not expressed the same during in vitro cultivation. Analyzing every individual peptide that is abundant in cultivation also ineffective and difficult ${ }^{(6)}$. Due to these problems, the immunoinformatics approach has been utilized to identify subunit vaccine candidates from bacterial, viral and parasite genome sequences. This approach called reverse vaccinology works by analyze the genome sequence in silico and predict the feasible peptide with the ability to act as an epitope and thus have the protective capacity ${ }^{(7)}$.

The first step of developing a peptide-based vaccine against PfEMP1 is to identify epitopes and predict its binding affinities to HLA alleles. A good vaccine candidate should be able to induce an adequate protective immune response against epitopes that are recognized by wide varieties of HLA alleles and generate cross-reaction with other isolates ${ }^{(8)}$. This study was conducted to identify T-cell and B-cell epitopes of the PfEMP1-DBL2 $\beta$ domain from Indonesian isolate using an immunoinformatic approach. The previous study reported that the PfEMP1-DBL2 $\beta$ recombinant protein is homolog with DBL $\beta$ PF11_0521 protein found in the cerebral malaria of Tanzania children. This protein is known to have an affinity with ICAM-1 receptor and estimated to have conserved epitopes, so it is considered as a candidate for a peptide-based malaria vaccine ${ }^{(9)}$. Identificatifying of T-cell and B-cell epitopes is crucial in designing the malaria vaccine because the protective immunity against malaria depends on the ability of both cells in generating immune responses ${ }^{(10)}$. Determination of the T-cell and B-cell epitopes of the recombinant protein will be useful for further study in developing a peptide-based vaccine.

\section{Method}

Analysis of protein hydrophobicity: The amino acid sequences of PfEMP1-DBL2 $\beta$ protein from Indonesian isolate cloned by Sulistyaningsih (9), which was deposited at the NCBI (https://www.ncbi.nlm.nih. gov/) with the accession number AGJ83325.1 were retrieved and subjected to check its hydrophobicity attribute by using Peptide Property Calculator Program (http://www.biosyn.com/PeptidePropertyCalculator/ PeptidePropertyCalculator.aspx).

\section{T-Cell Epitopes Prediction for MHC I and MHC}

II: The binding of antigen to major histocompatibility complex (MHC) molecules possess a significant role in determining whether an antigen is immunogenic or not. Prediction of the T-cell epitope in developing a subunit vaccine can diminish the experimental step to identify the suitable epitopes in vaccine design. In this study, T-cell epitope prediction for MHC I was done by using NetCTL (http://www.cbs.dtu.dk/services/NetCTL/), an online server for predicting epitopes of human cytotoxic $\mathrm{T}$ lymphocyte (CTL) based on protein sequence input. The epitope prediction was conducted by combining the prediction of MHC Class I affinity, TAP transport efficiency and proteasomal cleavage for $12 \mathrm{MHC}$ I supertypes. This tool has better predictive performance compared to several other tools in large scale data ${ }^{(11)}$. The threshold for $\mathrm{C}$ terminal cleavage was put on 0.15 , 0.05 for TAP transport efficiency and 0.75 for epitope prediction $^{(12)}$.

Identified T-cell epitopes for MHC-I were further evaluated for its antigenicity to predict the capacity in generating an immune response. The antigenicity prediction tool used in this study was the Vaxijen 2.0 online antigen prediction (http://www.ddg-pharmfac. 
net/vaxijen/VaxiJen/VaxiJen.html). This server was developed to predict protective antigens from several hosts and capable of classifying antigen based on its physicochemical properties, resulting in an antigen probability report for each protein. With the threshold set on 0.5 , this prediction server can perform with an accuracy of $87 \%^{(13)}$. T-cell epitopes for MHC I alleles with the highest probability as a protective antigen were subjected to conservancy analysis with IEDB tools (http://www.iedb.org/) with a 100\% threshold compared with Pf11_0521 sequences (accession number XP_001348176.1) and analyzed for its coverage withinpopulation by keeping the parameters on default. The population coverage analysis resource tool determines the ratio of individuals predicted to react toward a given set of epitopes.

Major histocompatibility class II (MHC Class II) is another major part in initiating an immune response. This molecule is normally found on antigen-presenting cells (APC) to present antigens derived from extracellular protein. Because MHC Class II interacts with other immune cells such as lymphocyte T helper cell (CD4+), identifying peptide that have an affinity with this molecule is important in vaccine design. Prediction of the binding peptide with MHC Class II was completed by using ProPred (https://webs.iiitd.edu.in/raghava/propred/), an online server to predict antigenic epitopes for 51 HLA Class II alleles based on the amino acid coefficient table. This server can locate the promiscuous binding region from the input protein. The default threshold was put on $3 \%$ to limit false-positive results ${ }^{(14)}$.

The affinity values for the predicted promiscuous peptides were evaluated using several tools available in the IEDB server. The NN-align algorithm was chosen due to its better predictive performance than other method for HLA-DR peptide binding prediction, such as SMM-align and NetMHCIIpan ${ }^{(15)}$. The predicted peptides were analyzed against three HLA-DR alleles from the malaria target population (Indonesian, East Africa and West Africa) chosen from Allele Frequency Net Database (http://www.allelefrequencies.net/default. asp) and the affinity was showed as IC50 values.

Identification of $\mathbf{B}$ cell epitope clusters: The B cell epitopes within PfEMP1-DBL2 $\beta$ were identified using two method, i.e., Bepipred 2.0 and KolaskarTangaonkar method in the IEDB server. Both method were chosen because of their accuracy. The Bepipred 2.0 is the latest epitope predictor that identify epitopes from peptide crystal structures. It was presumed to give higher quality prediction than other available tools ${ }^{(16)}$. Moreover, the Kolaskar-Tangaonkar method uses the physicochemical properties of amino acid and their frequencies of occurrence to predict protein antigenic determination $^{(17)}$. With default parameters, both method gave antigenicity scores as a result, which is categorized using the K-means clustering method and depicted in a heat map showed epitope densities of the amino acid sequence.

\section{Research Findings:}

Hydrophobicity Attribute Analysis: The PfEMP1-DBL2 $\beta$ protein was subjected to measure the hydrophobicity attribute by using the Peptide Property Calculator program. Protein with hydrophobicity $<50 \%$ is soluble in aqueous solution, while hydrophobicity $>50 \%$ is considered partially soluble or insoluble. The more soluble in the aqueous phase, the bigger the probability of the protein to fit into MHC molecules active site cleft ${ }^{(8)}$. Based on the Peptide property calculator program, the hydrophobicity value of PfEMP1-DBL2 $\beta$ protein was 32.62 , indicated that this protein is fully soluble in the aqueous phase.

T-Cell Epitopes Prediction: NetCTL prediction tool showed a total of 137 epitope sequences reacting with 12 MHC I alleles supertypes, with 31 sequences reacted with multiple supertypes (Supplementary Table 1). Each epitope sequence was analyzed for the antigenicity using VaxiJen 2.0 server, showing antigen probability classification. IEDB conservancy analysis tool further analyzes conserved sequences compared with Pf11_0521, a PfEMP1 protein isolated from Tanzania children. Two conserved T-cell epitopes from MHC I were generated, as listed in Table 1. Both conserved sequences met the criteria of the default threshold level in VaxiJen 2.0, which is $\geq 0.5$.

Table 1. Conserved sequences for T-cell epitopes.

\begin{tabular}{|l|c|c|}
\hline $\begin{array}{l}\text { T-cell Epitope } \\
\text { Sequences }\end{array}$ & Amino Acid Position & $\begin{array}{c}\text { Antigenicity } \\
\text { Score }\end{array}$ \\
\hline YIPQRLRWM & $219-227$ & 1.2417 \\
\hline WMTEWAEWY & $226-240$ & 0.7684 \\
\hline
\end{tabular}

Explanation: Antigenicity score, as predicted by VaxiJen 2.0 server $\geq 0.5$, showed that the peptides were classified as an antigen and able to induce host immune response. 
Population Coverage Analysis of MHC I Epitopes: Highly polymorphic MHC molecules remains a challenge for researchers in developing an effective vaccine. The high number of HLA allelic sequences identified were showed as a widely varying binding specificity of MHC molecules and expressed at different frequencies in different ethnicities. Because malaria is endemic to only several populations, choosing the suitable peptides that reacting with the most expressed
MHC alleles in target populations is necessary for rational vaccine design.

IEDB population coverage analysis tool calculates the percentage of individuals predicted to react with a given set of epitopes. Two conserved epitopes of MHC I molecules exhibited different coverage rates in several different populations known as the malaria-endemic area, as depicted in Figure 1.

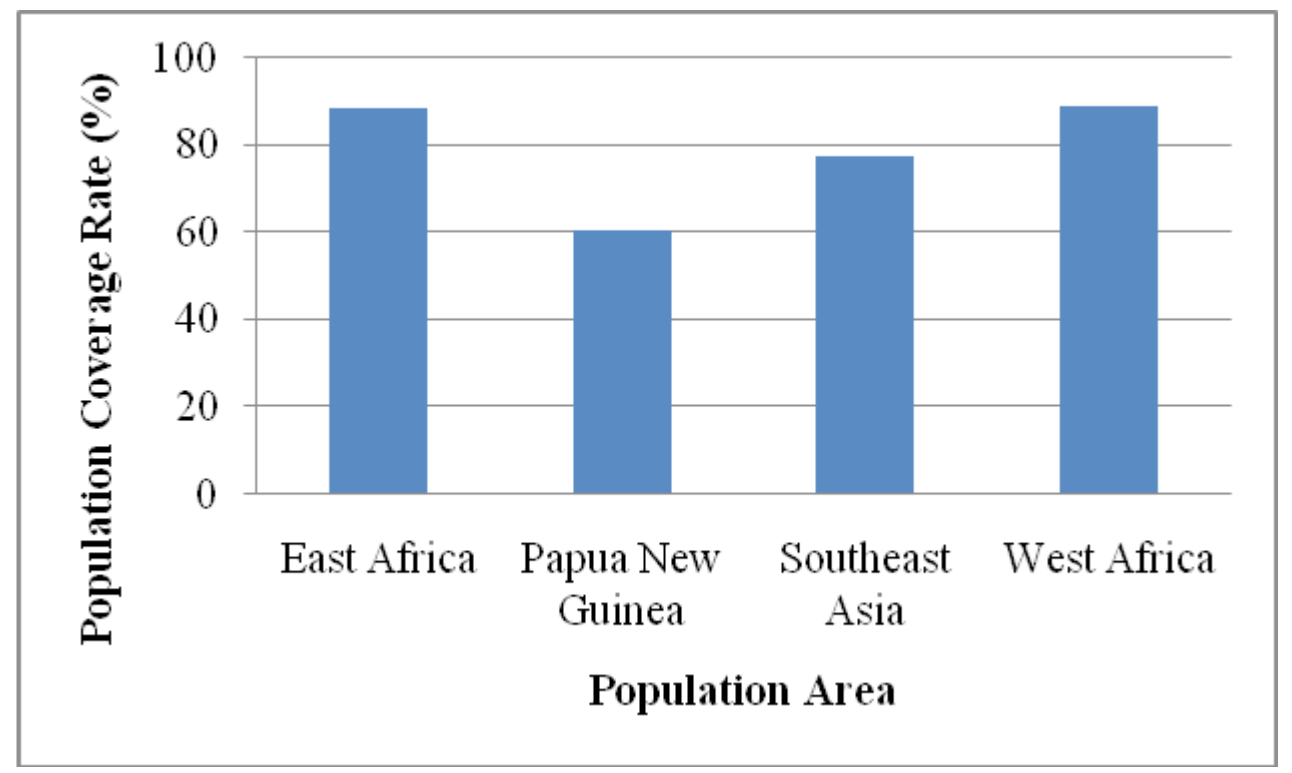

Figure 1: Predicted Population Coverage Rate (\%) of two conserved MHC I epitopes (YIPQRLRWM and WMTEWAEWY) of DBL2 $\beta-P f E M P 1$ recombinant protein Indonesian isolate in the malaria target population.

For epitope prediction based on its affinity with MHC II alleles, the Propred prediction generated 31 epitope sequences that act as promiscuous peptides (Supplementary Table 2). In the same manner with epitope prediction for MHC I allele, Vaxijen 2.0 server was used to analyze the antigenicity score and classify each peptide into an antigen and non-antigen, resulted in 17 epitope sequences predicted as antigen.
The prediction analysis of $\mathrm{T}$ cell epitopes affinity toward the MHC II allele used the $\mathrm{NN}$-align algorithm in IEDB ${ }^{(15)}$. The IC50 values distribution between each amino acid position was presented in Figure 2. Epitopes with scores lower than $50 \mathrm{~nm}$ were considered as strong binders, $50-500 \mathrm{nM}$ as intermediate binders and $>500$ $\mathrm{nM}$ as weak binders. Figure 2 also showed that the strong binding amino acid positions are different among the three target population HLA-DR alleles chosen in this study. 


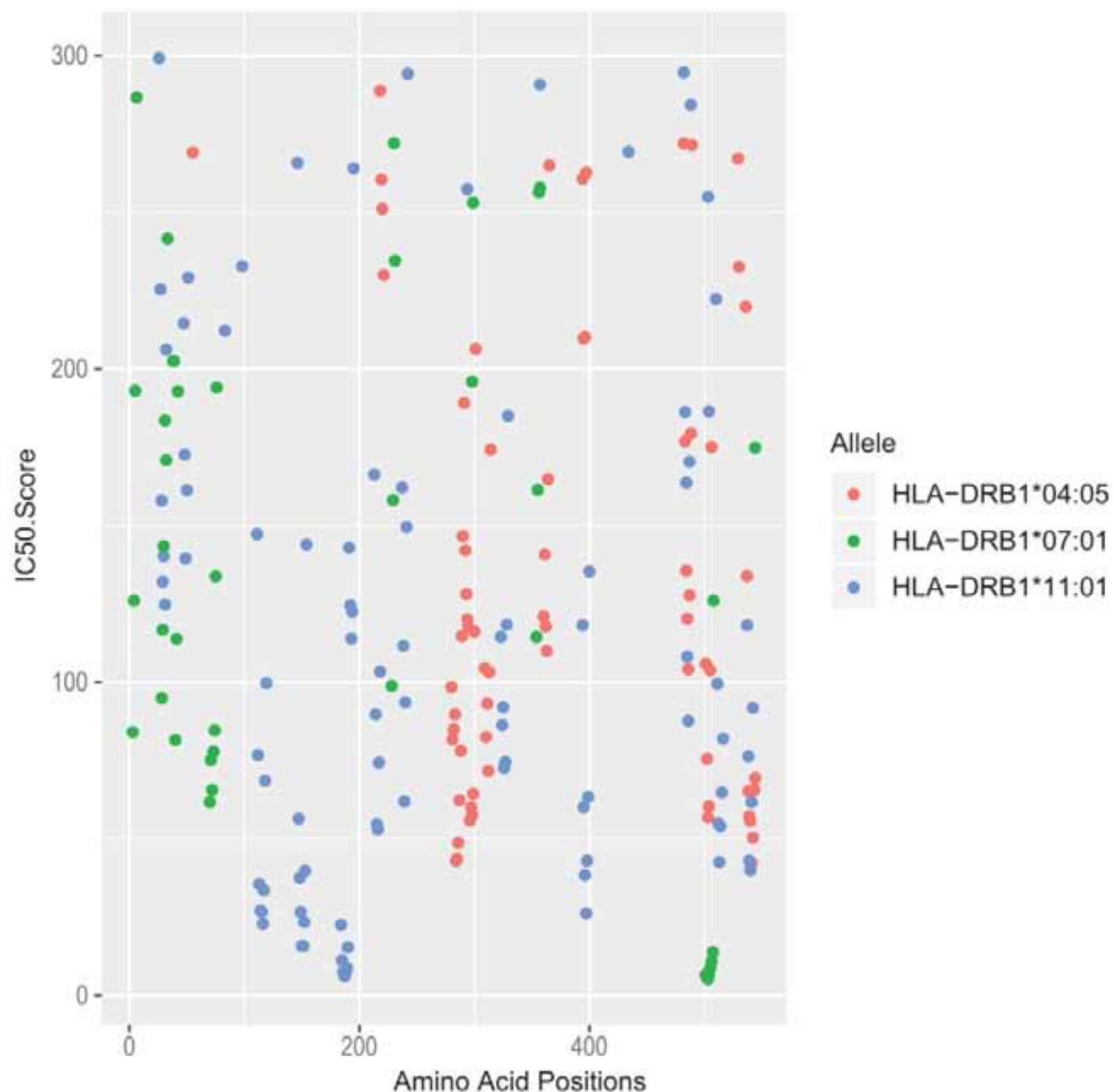

Figure 2: $\mathrm{IC}_{50}$ values at each amino acid position for Th cell epitopes in DBL2 $\beta$-PfEMP1 protein. The predictions were derived from the $\mathrm{NN}$-align algorithms for three alleles from Indonesia and Africa. Low $\mathrm{IC}_{50}$ indicates strong binding between epitopes and MHC Class II molecules.

B cell epitopes prediction in PfEMP1-DBL2 $\beta$ protein: Two techniques performed determination of the $B$ cell epitopes presence in PfEMP1-DBL2 $\beta$ protein, i.e., the Bepipred 2.0 algorithm $^{(16)}$ and Kolaskar-Tangaonkar method $^{(17)}$. Subsequently, the antigenicity scores and epitope density regions on each amino acids were used as a variable in the K-means clustering method to generate a heat map showed in Fig 3. This method has been used to differentiate regions with a higher concentration of epitopes $^{(18)}$. 


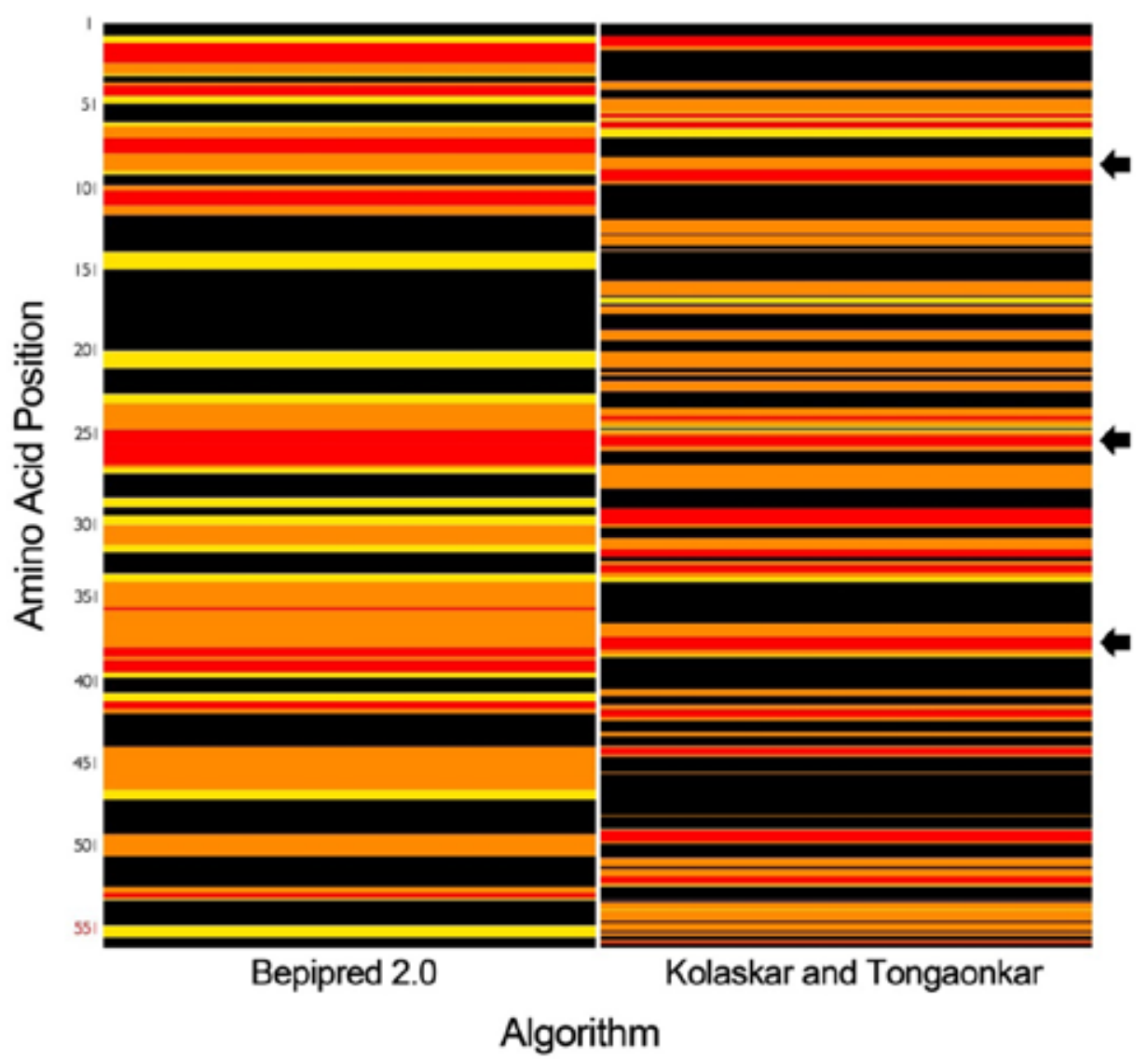

Figure 3: A heat map depicted the distribution of epitope dense regions throughout the DBL2及-PfEMP1 amino acid sequence. The regions were determined based on Bepipred 2.0 algorithm and KolaskarTangaonkar method, clustered by the K-means method. Each amino acid categorized into high, medium, low and non-epitope based on its antigenicity score. The red color represents a region with high antigenicity score epitope, orange indicates a region with medium antigenicity score epitope, yellow depicts region with low antigenicity score epitope and black denotes regions with no B-cell epitope. Potential B-cell epitope clusters are speculated positioned in the area with overlapping red and orange colors between two method (black arrows).

The Bepipred 2.0 algorithm predicted different epitope cluster regions from the Kolaskar-Tongaonkar method. We hypothesized that the overlapping position between two method as a potential B cell epitope clusters with maximum antigenicity score.
Within the 558 amino acid sequence designated in the heat map, the K-clustering method predicted several epitope clusters with the highest antigenicity, as shown in Table 2. One of these epitope clusters coincidentally aligned with the $\mathrm{Y}$ motif area of PfEMP1-DBL2 $\beta$ $\operatorname{protein}^{(19,20)}$.

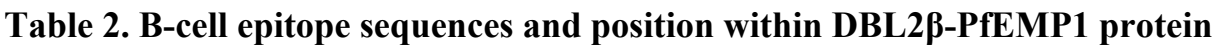

\begin{tabular}{|l|c|c|}
\hline B-cell epitope Sequences & Amino Acid Position & Area \\
\hline DTHTLLGEVALSA & $77-89$ & Semi-conserved area \\
\hline YQSQKYDELKKQCSQCKSK & $236-254$ & Semi-conserved area \\
\hline NTAAGYIHQELQQVGCNT & $360-377$ & Y motif, conserved area \\
\hline
\end{tabular}


Explanation: B-cell epitopes prediction in overlapping area and their position within DBL2 $\beta$ PfEMP1 protein. The first two epitopes located in the semi-conserved area and one aligned with the Y motif, which is a conserved area as well as a binding area with ICAM-1 receptor.

\section{Discussion}

The development of a malaria vaccine is still facing many obstacles. High antigenic switching rates and immune evasion ability of the parasites often cause a specific antigen-based vaccine not able to generate the expected immune response. Retrieving information about antigen immunogenicity through in vitro or in vivo experiments needs considerable time and resources, scientists call for another approach to obtain the information prior to the true experimental procedure, i.e., the bioinformatic approach, it is a powerful tool to analyze antigenic protein properties in order to design an efficient and effective vaccine ${ }^{(8)}$. In this study, an immunoinformatic approach towards PfEMP1-DBL2 $\beta$ protein from Indonesian isolate cloned previously by Sulistyaningsih ${ }^{(9)}$ was used to analyze the potency of the recombinant protein to bind to $\mathrm{T}$ cell and $\mathrm{B}$ cell lymphocyte.

The major histocompatibility complex (MHC) molecule is a group of receptors on the cell surface and has a major role in the immune response. Peptide sequences expressed on MHCs are called T Cell epitopes. Generally, MHC is classified into two classes; MHC I molecules with $\beta 2$ microglobulin subunit, which can be recognized by CD8 co-receptors (Cytotoxic T Cell/ CTL) and MHC II molecules with $\beta 1$ and $\beta 2$ subunit which can be recognized by CD4 co-receptors ( $\mathrm{T}$ helper cell/Th cell).

A part of the antigenic protein bind to MHC molecules that can be recognized by lymphocyte $\mathrm{T}$ cell and capable of inducing an immune reaction is called immune epitopes ${ }^{(21,22)}$. Total 31 MHC I epitopes predicted in this study were subjected to population coverage analysis, showed that two conserved MHC I epitopes from PfEMP1-DBL2 $\beta$ Indonesian isolate protein (YIPQRLRWM and WMTEWAEWY) have almost $90 \%$ coverage rate in East Africa and West Africa. The coverage rate indicates that a vaccine design based on these presumed epitopes might be performed efficiently for most of the population in East and West Afric, where the incidence of malaria is the highest ${ }^{(1)}$.
Using different algorithm predictions from the MHC I epitopes for CTL, the analysis for Th cell epitopes mediated by MHC II molecules, showed 17 promiscuous peptides predicted to be antigenic. The potency of PfEMP1-DBL2 $\beta$ protein from Indonesian isolate as a T-cell epitope-based subunit vaccine was analyzed by the $\mathrm{NN}$-align algorithm to identify its immune epitope. The study used three different HLA alleles commonly found in the Indonesian and African populations (HLA-DRB1*04:05, HLA-DRB1*07:01 and HLA-DRB $1 * 11: 01)$, there are several epitopes found with IC50 values under 50 . Even though hydrophobicity values of this protein showed that it potentially fits into the active site cleft of HLA alleles, epitopes mapping depicted in Fig 2 showed that there are no overlapping epitope positions for the three alleles with IC50 values under 50. It indicated that HLA alleles polymorphic variations still pose as a factor affecting PfEMP1-DBL2 $\beta$ protein from Indonesian isolate affinity toward MHC Class II molecules. Additional allele variants may allow broader population coverage and can be very useful in $\mathrm{T}$ lymphocyte cell epitope-based vaccine development ${ }^{(21)}$.

Aside from lymphocyte T cells' immune responseability, lymphocyte B cells also have some essential functions in specific antibody production. Peptide regions that bind to lymphocyte $\mathrm{B}$ cell receptors are called B cell epitopes. The epitopes analysis on lymphocyte B cell was done by using two algorithms, i.e., Bepipred 2.0 and Kolaskar-Tangaonkar Method. Both predictions showed the antigenicity scores for every amino acid composing PfEMP1-DBL2 $\beta$ protein from Indonesian isolate, which was subsequently categorized based on epitopes density area in the sequences and depicted as a heat map. Fig 3 showed different epitope clusters position between two prediction method in the heat map. Several overlapping clusters were observed at the position of 77-89, 236-254 and 360-377, implicated locations of B cell epitopes with the highest antigenicity and density.

An epitope located at the position of $360-377$ is coincidentally aligned with one of the few conserved areas in PfEMP1-DBL2 $\beta$ protein, called $\mathrm{Y}$ motif ${ }^{(19)}$. The $\mathrm{Y}$ motif is known as conserved residues that have functioned as a binding area with ICAM-1 receptor found in cerebral vasculatures. Binding between ICAM1 and PfEMP1-DBL2 $\beta$ domain via $\mathrm{Y}$ motif is essential in the pathogenesis of cerebral malaria ${ }^{(23,24)}$. A shared conserved epitope cluster is important in malaria vaccine development because, with the high antigenic switching rate of PfEMP1, this cluster can be recognized by the 
immune system and potentially able to generate a crossreaction between parasite isolates ${ }^{(25,26)}$. Several studies showed that cross-reaction between PfEMP1 isolates do exist, P. falciparum infection in tourists who just come back from an endemic area have antibody with wide cross-reaction and persistent for more than 20 weeks post-infection $^{(27,28)}$. This phenomenon, also found in placental malaria, mediated by var2CSA, showed polymorphism but wide cross-reaction toward different isolates $^{(29)}$. The molecular base for this cross-reaction toward specific antigen has not been fully understood, but polymorphic shared epitopes between PfEMP1 isolates are expected to have a major role $\mathrm{e}^{(4,30)}$.

\section{Conclusion}

The vaccine development to combat malaria as a global health problem is an important issue. In addition to advancements in technology, performing in silico study through an effective and efficient immunoinformatic approach provides ease in analyzing protein immunogenicity prior to in vitro or in vivo experimental tests for vaccine development. In this current study, the immunogenicity value of PfEMP1-DBL $2 \beta$ protein from Indonesian isolate was conducted by Th cell and B cell epitope predictions. Homolog with DBLPPF11_0521 protein found in Tanzania children, the PfEMP1 from Indonesian isolate has B cell epitopes located in the conserved $\mathrm{Y}$ motif area, making it a potential subunit peptide-based malaria vaccine. A further experimental study concentrated on the delivery mechanisms and the in vitro or in vivo experimental of interaction with the immune system is needed to validate this approach as the next step in subunit peptide-based vaccine development.

Conflict of Interest: The authors have no conflict of interest to declare.

Source of Funding: This research was financially supported by the Islamic Development Bank (IsDB) through Four in One Project 2017-2019.

Ethical Clearance: Ethical approval with the reference number 454/H25.1.11/KE/2014 was obtained from The Ethical Committee of Faculty of Medicine, University of Jember, Indonesia.

Consent: The written informed consent was obtained from all the study participants. The Indonesian Plasmodium falciparum isolate was isolated from the blood of malaria patients. The patients were explained about the study and signed consent after a detailed explanation.

\section{References}

1. WHO. World Malaria Report 2018.; 2018.

2. Laurens MB. The Promise of a Malaria Vaccine-Are We Closer? Annu Rev Microbiol. 2018;72(September 2018):273-292.

3. Schofield L, Grau GE. Immunological processes in malaria pathogenesis. Immunol Nat. 2005;5(22):9196. doi:10.1038/nri1686

4. Chan J-A, Fowkes FJI, Beeson JG. Surface antigens of Plasmodium falciparum-infected erythrocytes as immune targets and malaria vaccine candidates. Cell Mol Life Sci. 2014;71(19):3633-3657. doi:10.1007/s00018-014-1614-3

5. Janes JH, Wang CP, Levin-edens, et al. Investigating the Host Binding Signature on the Plasmodium falciparum PfEMP1 Protein Family. PLoS Pathog. 2011;7(5). doi:10.1371/journal.ppat.1002032

6. Doytchinova IA, Flower DR. Identifying candidate subunit vaccines using an alignment-independent method based on principal amino acid properties. Vaccine. 2007;25(5):856-866. doi:10.1016/j. vaccine. 2006.09.032

7. Mora M, Veggi D, Santini L, Pizza M, Rappuoli R. Reverse vaccinology. Drug Discov Today. 2003;8(10):459-464. doi:10.1016/S13596446(03)02689-8

8. Khan N, Kumar R, Chauhan S, Farooq U. An immunoinformatics approach to promiscuous peptides design for Plasmodium falciparum erythrocyte membrane protein-1. Mol Biosyst. 2017. doi:10.1039/C7MB00332C

9. Sulistyaningsih E, Hidayah F, Prasetyo A. Cloning and protein structure prediction of DBL2 $\beta$-PfEMP1 recombinant protein from Indonesian Plasmodium falciparum isolate. Curr Top Pept Protein Res. 2018;19(1):75-79.

10. Horata N, Choowongkomon K, Ratanabunyong S, Tongshoob J, Khusmith S. Acquisition of naturally acquired antibody response to Plasmodium falciparum erythrocyte membrane protein 1-DBL $\alpha$ and differential regulation of $\operatorname{IgG}$ subclasses in severe and uncomplicated malaria. Asian Pac J Trop Biomed. 2017;7(12):1055-1061. doi:10.1016/j. apjtb.2017.09.017

11. Larsen M V., Lundegaard C, Lamberth K, Buus $\mathrm{S}$, Lund $\mathrm{O}$, Nielsen M. Large-scale validation of method for cytotoxic T-lymphocyte epitope 
prediction. BMC Bioinformatics. 2007;8:1-12. doi:10.1186/1471-2105-8-424

12. Nielsen M, Lundegaard C, Worning P, et al. Reliable prediction of T-cell epitopes using neural networks with novel sequence representations. Protein Sci. 2003;12:1007-1017. doi:10.1110/ps.0239403.view

13. Srivastava PN, Jain R, Dubey SD, Bhatnagar S, Ahmad N. Prediction of Epitope-Based Peptides for Vaccine Development from Coat Proteins GP2 and VP24 of Ebola Virus Using Immunoinformatics. Int J Pept Res Ther. 2016;22(1):119-133. doi:10.1007/ s10989-015-9492-6

14. Singh H, Raghava GPS. ProPred: Prediction of HLA-DR binding sites. Bioinformatics. 2001;17(12):1236-1237. doi:10.1093/ bioinformatics/17.12.1236

15. Nielsen $M$, Lund $O$. NN-align. An artificial neural network-based alignment algorithm for MHC class II peptide binding prediction Morten. BMC Bioinformatics. 2009;10(296):1-10. doi:10.1186/1471-2105-10-296

16. Jespersen MC, Peters B, Nielsen M, Marcatili P. BepiPred-2.0: improving sequence-based B-cell epitope prediction using conformational epitopes. Nucleic Acids Res. 2017;45(May):24-29. doi:10.1093/nar/gkx346

17. Kolaskar AS, Tongaonkar PC. A semi-empirical method for prediction of antigenic determinants protein antigens. 1990;276(1):172-174.

18. Islam SMT, Zaman S, Khan MK, et al. MultiEpitope Cluster Ep85B within the Mycobacterial Protein Ag85B Elicits Cell-Mediated and Humoral Responses in Mice. Turkish J Immunol. 2018;6(3):108-117.

19. Oleinikov A V, Amos E, Frye IT, et al. High Throughput Functional Assays of the Variant Antigen PfEMP1 Reveal a Single Domain in the 3D7 Plasmodium falciparum Genome that Binds ICAM1 with High Affinity and Is Targeted by Naturally Acquired Neutralizing Antibodies. PLOS Pathog. 2009;5(4). doi:10.1371/journal. ppat. 1000386

20. Howell DP, Levin EA, Springer AL, et al. Mapping a common interaction site used by Plasmodium falciparum Duffy binding-like domains to bind diverse host receptors. Mol Microbiol. 2008;67(November 2007):78-87. doi:10.1111/ j.1365-2958.2007.06019.x
21. Wang P, Sidney J, Kim Y, et al. Peptide binding predictions for HLA DR, DP and DQ molecules. BMC Bioinformatics. 2010;11(568):1-12.

22. Abbas AK, Lichtman AH, Pillai S. Cellular and Molecular Immunology Eight Edition. Eight Edit. (Merritt J, ed.). Canada: Elsevier Inc. Saunders; 2015.

23. Springer AL, Smith LM, Mackaya DQ, Nelson SO, Smith JD. Functional interdependence of the DBL $\beta$ domain and $c 2$ region for binding of the Plasmodium falciparum variant antigen to ICAM1. Mol Biochem Parasitol. 2004;137(May):55-64.

24. Sulistyaningsih E, Fitri LE, Löscher T, Berens-riha N. Diversity of the var gene family of Indonesian Plasmodium falciparum isolates. Malar J. 2013; 12(1):80-93.

25. Rask TS, Hansen DA, Theander TG, Pedersen AG, Lavstsen T. Plasmodium falciparum Erythrocyte Membrane Protein 1 Diversity in Seven Genomes Divide and Conquer. PIOS Comput Biol. 2010;6(9). doi:10.1371/journal.pcbi.1000933

26. Bengtsson A, Joergensen L, Rask TS, et al. A Novel Domain Cassette Identifies Plasmodium falciparum PfEMP1 Proteins Binding ICAM-1 and Is a Target of Cross-Reactive, Adhesion-Inhibitory of Antibodies. J Immunol. 2012. doi:10.4049/ jimmunol.1202578

27. Elliott SR, Spurck TP, Dodin JM, et al. Inhibition of Dendritic Cell Maturation by Malaria Is Dose Dependent and Does Not Require Plasmodium falciparum Erythrocyte Membrane Protein 1. Infect Immun. 2007;75(7):3621-3632. doi:10.1128/ IAI.00095-07

28. Dara A, Travassos MA, Adams M, et al. A new method for sequencing the hypervariable Plasmodium falciparum gene var2csa from clinical samples. Malar J. 2017:1-9. doi:10.1186/s12936017-1976-8

29. Beeson JG, Mann EJ, Byrne TJ, et al. Antigenic Differences and Conservation among Placental Plasmodium falciparum - Infected Erythrocytes and Acquisition of Variant-Specific and Cross-Reactive Antibodies. J Infect Dis. 2006;193(5):721-730.

30. Hommel M, Elliott SR, Soma V, et al. Evaluation of the Antigenic Diversity of Placenta-Binding Plasmodium falciparum Variants and the Antibody Repertoire among Pregnant Women $\square \dagger$. Infect Immun. 2010; 78(5):1963-1978. doi:10.1128/ IAI.01365-09 